\title{
Mariposas diurnas y nocturnas \\ (Lepidoptera: Papilionoidae, Saturniidae, Sphingidae) del Parque Nacional Natural Gorgona, Pacífico Colombiano
}

\author{
Humberto Calero-Mejía ${ }^{1 *}$, Inge Armbrecht ${ }^{1}$ \& James Montoya-Lerma ${ }^{1}$ \\ 1. Departamento de Biología, Universidad del Valle, Calle 13 No 13-00 Cali, Colombia; \\ humcame@gmail.com, inge.armbrecht@correounivalle.edu.co,james.montoya@correounivalle.edu.co
}

Recibido 18-X-2013. Corregido 20-XI-2013. Aceptado 19-XII-2013.

\begin{abstract}
Butterflies and moths (Lepidoptera: Papilionoidae, Saturniidae, Sphingidae) at the Natural National Park Gorgona, Colombian Pacific. Gorgona is a continental island at the Pacific coast of Colombia. For 26 years it was a prison but in 1986 it was declared a National Park, and the land was left aside for natural succession. This study presents a list of its Lepidoptera, compares it to a former study and discusses some ecological factors that could promote in situ biodiversity. Butterflies and moths were collected during three trips from October 2010 to May 2011. Butterfly observations were carried out along the main island paths. Baited traps using decomposed fruit, fish and chicken, were installed for three days at each zone (two kinds of bait per site) and sweep net catches were also made between 09:00h and 18:00h. For moths, black and white light traps were located in open areas. Town lights were also checked daily. Percent canopy cover was measured at each collection point. A total of 52 species belonging to seven families were found, of which 30 butterfly and 11 moth species are new records for the island. Cerro Trinidad and Sendero Chonta (22 and 20 species, respectively) were the richest places. Species richness did not decrease with canopy cover. Heliconious sara and Aeria eurimedia were the dominant species, but their distribution did not overlap. Three species were the most widely distributed in the island: H. sara (11 sites, 166 individuals), Calycopis cerata (10 sites, 71 individuals) and Ae. eurimedia (nine sites, 72 individuals). Catoblepia xanthicles occidentalis was found 87 years after the last report. Most species accumulation curves were not asymptotic suggesting that the diversity of the island is higher than expected. This study found twice as much the number of butterflies species reported for Gorgona and is the first record of moths for the island. Rev. Biol. Trop. 62 (Suppl. 1): 317-328. Epub 2014 February 01.
\end{abstract}

Keys words: Colombia, butterflies, inventory, moths, national park, island.

La información proveniente de inventarios biológicos permite evaluar el estado actual de un taxón foco, analizar el estado ecológico de hábitats objeto de conservación, identificar especies vulnerables o que pueden estar en peligro de extinción y aportar a datos de distribución y biología de las especies (Kattán \& Naranjo, 2008). Entre los insectos, las mariposas son uno de los grupos ideales para inventarios rápidos de biodiversidad (Brown, 1991). Entre otras características, su relativa facilidad de identificación, sus amplios estudios taxonómicos y biológicos, respuestas rápidas a los cambios medioambientales y su carácter carismático, facilitan los trabajos en pro de la conservación de sus hábitats (Villareal et al. 2004).

Por mucho tiempo, el conocimiento de la fauna, en particular la entomofauna del Parque Nacional Natural Gorgona (PNNG) se ha basado en muestreos generales, descripción de nuevos taxones (Aldrete, González \& Sarria, 2011), distribución (Baena, 1992) y abundancia (Gómez, 2009; González \& Sarria, 2010; Pimienta, Montealegre, López-Mejía, Chacón de Ulloa \& González-Obando, 2008; Torres, Pinilla \& Chamorro, 1988). Sobre Lepidoptera 
sólo se conocen los trabajos de Andrade (1990), que registró 13 especies y Salazar, Rodríguez \& Constantino (2009) quienes en una revisión del género Mesosemia describieron M. zonalis gorgoniensis como una subespecie endémica.

Posterior a la declaratoria de área protegida de la isla Gorgona, por muchas décadas prisión de alta seguridad (1959-1985), se inició un proceso de sucesión vegetal desde el momento en que se redujo la presión antropogénica, y esto cambió la composición de la biota local y las interacciones ecológicas, las cuales se hacen más complejas (Green, Sadedin \& Leishman, 2009). En el caso particular de lepidópteros, si la composición vegetal cambia, la nueva distribución y abundancia de recursos alimenticios y microhábitats serán determinantes en el establecimiento y persistencia de los lepidópteros en la isla (e.g. Gilbert, 1980). Además, Gorgona es una isla de origen volcánico, posiblemente la continuidad de una cuarta cordillera que existió en el Mioceno (Baena \& Alberico, 1991) y puede albergar especies de alta rareza biogeográfica, endemismos u organismos vulnerables (Amat, Andrade \& Amat, 2007).

Este estudio, enmarcado en el proyecto: "Evaluación del estado actual de los objetos de conservación faunísticos en Isla Gorgona: una aproximación holística a la valoración ecológica de PNN Gorgona" y está enfocado a actualizar el listado de las especies de lepidópteros Papilionoidea, hacer la primera aproximación de polillas Saturniidae, Sphingidae, y describir la distribución espacial y preferencias ecológicas. Considerando la hipótesis de perturbación intermedia, se predice que la riqueza de lepidópteros aumentará en hábitats en estados sucesionales intermedios en isla Gorgona.

\section{MATERIALES Y MÉTODOS}

Área de estudio: Los sitios de muestreos abarcaron aproximadamente $65 \%$ del área terrestre del PNN Gorgona ( $2^{\circ} 47^{\prime}-3^{\circ} 6^{\prime}$ $\mathrm{N}$ y $78^{\circ} 6^{\prime}-78^{\circ} 18^{\prime} \mathrm{W}$ ) visitando los lugares con acceso seguro. Las zonas de muestreo se categorizaron en tres grupos por su estado de conservación. Para establecer las categorías se consideró la cercanía al poblado y los se tuvieron en cuenta los impactos al entorno generados en el pasado y las actividades actuales de ecoturismo. En este sentido se definieron como: (1) sitios: de perturbación alta: sendero La Chonta (SC) y el Poblado $(\mathrm{Pb})$, donde la presión antrópica es constante; (2) sitios: con perturbación media: senderos de Playa Yundigua (PY), Playa Blanca (PB), El Acueducto (Ac); y (3) sitios: con menor perturbación: Playa Palmeras (PP) El Mirador (Md), La Camaronera $(\mathrm{Cm})$, Playa Mancora (Mc), Gorgonilla (Gll), Cerro Trinidad (CT) y El Horno (Hr) donde la perturbación es casi nula, pero no significa que toda la vegetación sea bosque primario.

Método de recolecta y registro: Se hicieron tres salidas de campo en octubre 2010 (siete días), febrero 2011 (16 días) y mayo 2011 (13 días). Se recolectaron mariposas utilizando los métodos descritos por Villareal et al. (2004) utilizando (a) seis trampas Van Someren-Rydon (VSR) cebadas con fruta fermentada (dos), con pescado (dos) y pollo descompuesto (dos), montadas en cada sitio durante tres días, y (b) observación directa y recolecta con red entomológica en trayectos de longitud no definida entre las 09:00 y 18:00h, con un esfuerzo de muestreo diario de 9 horas/hombre. Para las polillas, se ubicaron en zonas abiertas, trampas de luz negra y blanca, reflejadas en una sábana de color blanco para maximizar la atracción en los senderos de Playa Blanca, Sendero La Chonta y Cerro Trinidad. Además, se revisaron las diferentes fuentes de luz artificiales del Poblado y la estación de buceo.

En cada punto de recolecta o registro se tomaron datos de altitud, coordenadas geográficas y porcentaje de cobertura vegetal de dosel utilizando un densiómetro esférico (Forestry Suppliers $\left.{ }^{\circledR}\right)$. Además, se registraron las interacciones de las mariposas con su ambiente, como hospederos, etología, entre otras.

Material recolectado: Los especímenes diurnos se sacrificaron mediante presión en el tórax y los nocturnos en cámara de acetato de etilo. Cada ejemplar fue guardado en 
sobres de papel mantequilla con silica gel y se determinaron hasta la menor categoría taxonómica posible siguiendo la clasificación de Lamas (2004), además de publicaciones y guías (Le Crom, Constantino \& Salazar, 2002; Valencia, Gil \& Constantino, 2005; D'Abrera (1987, 1988; 1994; 1995), catálogos en línea: Gema Virtual (2007), Warren, Davis, Grishin, Pelham \& Stangeland (2011), Kitching et al. (2009), Oehlke (2012). Se contó con la confirmación de los especialistas B. Oehlke (Sphingidae), C. Ríos-Malaver (Lycaenidae), H. Danners (Nymphalidae, Lycaenidae, Riodinidae), K. Willmot (Ithomiinae) y A. Amarillo (Saturniidae). Los ejemplares se depositaron en el Museo de Entomología de la Universidad del Valle (MUSENUV, GOR-4116-4305) en Cali-Colombia.

Análisis de datos: Playa Mancora, Camaronera, Gorgonilla y El Horno fueron visitados una o dos veces, por tal motivo solo se tienen en cuenta para el reporte de distribución espacial. Los análisis numéricos solo se realizaron para las mariposas, debido al bajo registro de polillas. Para cada sitio se obtuvo una matriz de presencia-ausencia y se calculó el número de especies esperadas mediante estimadores no paramétricos (Chao 2, Jackknife 1 y 2), los cuales permiten un mejor análisis con menor sesgo, debido a que tiene en cuenta datos de presencia-ausencia y de muestras únicas o escasas (Prieto \& Dahners, 2006). Además se calcularon los índices de diversidad de Shannon y equitabilidad. Con las coordenadas geográficas se realizó un mapa de distribución para las especies más abundantes y endémicas. El porcentaje de cobertura de dosel se agrupó en cinco categorías $(0-20 ; 21-40 ; 41-60 ; 61-80$ y 81-100) y se relacionó con la riqueza de lepidópteros. Estos datos se sometieron a la prueba de correlación de Spearman. Se realizó un análisis de composición de las especies, mediante aglomeración utilizando el coeficiente de similitud de Jaccard con algoritmo de grupos pareados y se realizó un análisis de similitud multivariado (ANOSIM) de una vía con el programa PAST (Hammer, Harper \& Ryan, 2008).

\section{RESULTADOS}

Riqueza y composición de especies: se recolectaron 146 individuos (41 especies diurnas y 11 nocturnas), divididas en siete familias y 18 subfamilias de las cuales se reportan 41 nuevos registros de especies (30 diurnas y 11 nocturnas). Las familias con mayor riqueza fueron Nymphalidae (8 subfamilias; 19 especies) y Riodinidae (2 subfamilias, 12 especies); las de menor riqueza fueron Pieridae (3 especies), Papilionidae (2 especies) y Lycaenidae

CUADRO 1

Listado de lepidópteros (Lepidoptera: Papilionoidea) del PNN Gorgona con sus distribuciones y algunos datos ecológicos

TABLE 1

Checklist of lepidopteron (Lepidoptera: Papilionoidea) of PNN Gorgona and their distribution and some ecology data

\begin{tabular}{|c|c|c|c|}
\hline Taxón & Distribución & Elevación & $\% \mathrm{CVD}$ \\
\hline \multicolumn{4}{|l|}{ Nymphalidae } \\
\hline \multicolumn{4}{|l|}{ Brassolinae } \\
\hline Catoblepia xanthicles occidentalis & Ac, PY, PB, Mc & $19-35 \mathrm{~m}$ & $97-98$ \\
\hline \multicolumn{4}{|l|}{ Charaxinae } \\
\hline Archaeoprepona demophon muson (Fruhstorfer, 1905) & $\mathrm{Pb}, \mathrm{CT}, \mathrm{SC}, \mathrm{PB}, \mathrm{Cm}$ & $4-36 \mathrm{~m}$ & 80 \\
\hline Prepona laertes (Hübner, [1811]) & Ac, CT, SC, Md & $27-90 \mathrm{~m}$ & - \\
\hline \multicolumn{4}{|l|}{ Danainae } \\
\hline Danaus plexippus (Linnaeus, 1758) & $\mathrm{Pb}$ & 27 & - \\
\hline \multicolumn{4}{|l|}{ Ithomiinae } \\
\hline Aeria eurimedia* & Todos menos PY, Pb, Gll & $13-246 \mathrm{~m}$ & $86-100$ \\
\hline
\end{tabular}


CUADRO 1 (Continuación) / TABLE 1 (Continued)

\begin{tabular}{|c|c|c|c|}
\hline Taxón & Distribución & Elevación & $\%$ CVD \\
\hline Oleria amalda $*$ (Hewitson, [1857]) & Ac, PY, CT, SC, PB, Md, Mc & $10-276 \mathrm{~m}$ & $86-100$ \\
\hline \multicolumn{4}{|l|}{ Morphinae } \\
\hline Morpho sp. & CT, PB & - & - \\
\hline \multicolumn{4}{|l|}{ Nymphalinae } \\
\hline Anartia jathrophae (Linnaeus, 1763) & $\mathrm{Pb}$ & $27 \mathrm{~m}$ & 4 \\
\hline Colobura. Annulata (Willmott, Constantino \& Hall, 2001) & PY, CT & $6-169 m$ & - \\
\hline Dynamine postverta mexicana D’Almeida, 1952 & Ac & $102 \mathrm{~m}$ & 98 \\
\hline Eunica sydonia (Godart [1824]) & $\mathrm{SC}$ & $30 \mathrm{~m}$ & - \\
\hline Eunica mygdonia (Godart [1824]) & $\mathrm{CT}$ & $338 \mathrm{~m}$ & - \\
\hline Smyrna blomfildia (Fabricius, 1781) & PY, CT & $6-84 \mathrm{~m}$ & 83 \\
\hline \multicolumn{4}{|l|}{ Heliconiinae } \\
\hline Dryas iulia* (Fabricius 1775) & $\mathrm{Pb}, \mathrm{SC}, \mathrm{PB}, \mathrm{PP}$ & $3-36 \mathrm{~m}$ & $0-97$ \\
\hline Heliconius cydno zelinde Butler, 1869 & $\mathrm{Ac}, \mathrm{CT}, \mathrm{SC}, \mathrm{Mr}, \mathrm{Hr}, \mathrm{Mc}$ & $15-216 \mathrm{~m}$ & $38-100$ \\
\hline Heliconius sara* (Fabricius, 1793) & Todos excepto Ac & $0-236 \mathrm{~m}$ & $0-100$ \\
\hline Euides isabella (Stoll, 1781) & $\mathrm{Pb}$ & $27 \mathrm{~m}$ & 4 \\
\hline \multicolumn{4}{|l|}{ Satyrinae } \\
\hline Hermeuptychia hermes (Fabricius, 1775) & $\mathrm{Py}, \mathrm{Pb}, \mathrm{CT}, \mathrm{SC}$ & $13-29 m$ & $63-98$ \\
\hline Magneuptychia libye* (Linnaeus, 1767) & $\mathrm{SC}, \mathrm{PB}$ & $14-35 \mathrm{~m}$ & $51-97$ \\
\hline \multicolumn{4}{|l|}{ Papilionidae } \\
\hline \multicolumn{4}{|l|}{ Papilioninae } \\
\hline Battus ingenuus (Dyar, 1907) & Ac, PY, SC, PB & $4-30 \mathrm{~m}$ & $77-97$ \\
\hline Parides sesostris tarquinius* (Boisduval, 1836) & Ac, PY, CT, SC, PB, Hr & $15-230 \mathrm{~m}$ & $88-100$ \\
\hline Taxón & Distribución & Elevación & $\% \mathrm{CVD}$ \\
\hline \multicolumn{4}{|l|}{ Lycaenidae } \\
\hline \multicolumn{4}{|l|}{ Polyommatinae } \\
\hline Hemiargus hanno* (Stoll, 1790) & PY & $35 \mathrm{~m}$ & 58 \\
\hline \multicolumn{4}{|l|}{ Theclinae } \\
\hline Calycopis cerata* (Hewitson, 1877) & Todos menos $\mathrm{Pb}, \mathrm{Cm}$ & $3-221 \mathrm{~m}$ & $36-100$ \\
\hline Celmia celmus (Cramer, 1775) & Ac, PY, CT, SC & $10-65 \mathrm{~m}$ & $91-100$ \\
\hline Lamprospilus collucia (Hewitson, 1877) & $\mathrm{CT}, \mathrm{SC}, \mathrm{PB}$ & $17-338 \mathrm{~m}$ & $94-97$ \\
\hline Ziegleria hesperitis (Butler \& H. Druce, 1872) & $\mathrm{Pb}, \mathrm{SC}$ & $4-27 \mathrm{~m}$ & 99 \\
\hline \multicolumn{4}{|l|}{ Pieridae } \\
\hline \multicolumn{4}{|l|}{ Dismorphinae } \\
\hline Dismorphia theucharila avonia* (Hewitson, 1867) & Ac, PY, CT, SC, PB, PP, Mr, Hr & $11-320 \mathrm{~m}$ & $92-100$ \\
\hline \multicolumn{4}{|l|}{ Coliadinae } \\
\hline Anteos mennipe (Hübner 1816) & $\mathrm{Ac}, \mathrm{Pb}, \mathrm{PB}$ & $15-89 \mathrm{~m}$ & 11 \\
\hline Phoebis argante larra (Fabricius, 1798) & $\mathrm{PY}, \mathrm{Pb}$ & $15 \mathrm{~m}$ & 54 \\
\hline \multicolumn{4}{|l|}{ Riodinidae } \\
\hline \multicolumn{4}{|l|}{ Euselasiinae } \\
\hline Euselasia eugeon (Hewitson, 1856) & $\mathrm{PY}, \mathrm{SC}, \mathrm{Mc}$ & $30-56 \mathrm{~m}$ & $91-100$ \\
\hline Euselasia sp.1 & $\mathrm{CT}, \mathrm{PB}, \mathrm{PP}, \mathrm{Cm}$ & $10-60 \mathrm{~m}$ & $85-100$ \\
\hline \multicolumn{4}{|l|}{ Riodininae } \\
\hline Calospila byzeres (Hewitson, 1872) & $\mathrm{PP}$ & $1 \mathrm{~m}$ & 31 \\
\hline Calospila idmon (Godman \& Salvin, 1889) & $\mathrm{PB}$ & $64 \mathrm{~m}$ & 95 \\
\hline Hypophylla argenissa (Stoll, 1790) & PY, CT & $145-239 m$ & 95 \\
\hline Mesosemia zonalis gorgoniensis* & $\mathrm{Ac}, \mathrm{PY}, \mathrm{Pb}$ & $16-89 \mathrm{~m}$ & $80-100$ \\
\hline Napaea eucharila (H. Bates, 1867) & CT, SC, Gll & $15-162 \mathrm{~m}$ & $91-100$ \\
\hline
\end{tabular}


CUADRO 1 (Continuación) / TABLE 1 (Continued)

\begin{tabular}{lccc}
\multicolumn{1}{c}{ Taxón } & Distribución & Elevación & \% CVD \\
Pixus corculum (Stichel, 1929) & CT, PY & $37-165 \mathrm{~m}$ & $85-95$ \\
Setabis myrtis (Westwood, 1851) & CT, PP, Mr & $28-314 \mathrm{~m}$ & $92-99$ \\
Synargis calyce (Felder \& Felder 1862) & PY, CT, PB & $16-234 \mathrm{~m}$ & - \\
Theope publius (Felder, 1861) & SC, PB, PP, Gll & $17-78 \mathrm{~m}$ & $89-100$ \\
Theope barea Godman \& Salvin, 1878 & Mc & $49 \mathrm{~m}$ & - \\
\hline
\end{tabular}

$\% \mathrm{CVD}=$ Porcentaje de cobertura de dosel, *Especies reportadas por Andrade, 1990.

(5 especies) (Cuadro 1). Respecto a las polillas, de Sphingidae se recolectaron 10 especies y se reporta Rothschildia lebeau (Guérin-Méneville, 1868) para Saturniidae (Cuadro 2). Se destaca la captura de una nueva subespecie de Oleria amalda (K. Willmot, com. pers. agosto 19,2010$)$ y la recaptura de Cat. xanthicles occidentalis (Fig. 1A), la cual había sido recolectado en isla Gorgona por última vez en 1924 durante la expedición "The Saint George
Expedition" (1924-1925) (P. Blandin, com. pers. octubre 15, 2013).

Los datos de los índices de diversidad y equitabilidad fueron altos tanto en sitios perturbados como menos perturbados (Cuadro 3 ). Para todas las especies diurnas el estimador Chao-2 fue de 42.5 y de Jacknife-2 de 48.8 lo que significa que la eficiencia de muestreo fue de 96.4 y $84 \%$, respectivamente. La asociación entre riqueza de mariposas y cobertura vegetal

\section{CUADRO 2}

Listado de polillas (Saturniidae, Sphingidae) del PNNG con la distribución por departamentos en Colombia y los nuevos reportes

TABLE 2

List of months (Saturniidae, Sphingidae) of PNNG with the department distribution in Colombia and new reports

\begin{tabular}{|c|c|c|}
\hline \multirow{2}{*}{ Taxón } & \multicolumn{2}{|c|}{ Distribución } \\
\hline & Previa & Ampliación \\
\hline \multicolumn{3}{|l|}{ Saturniidae } \\
\hline \multicolumn{3}{|l|}{ Saturniinae } \\
\hline Rothschildia lebeau & Ant, VC, Snt, Met & $\mathrm{Cc}(\mathrm{PNNG})$ \\
\hline \multicolumn{3}{|l|}{ Sphingidae } \\
\hline \multicolumn{3}{|l|}{ Macroglossinae } \\
\hline Aellopos titan (Cramer, 1777) & Met, Ant & $\mathrm{Cc}(\mathrm{PNNG})$ \\
\hline Calionima nomius (Walker, 1856) & Ant & $\mathrm{Cc}(\mathrm{PNNG})$ \\
\hline Callionima pan (Cramer, 1779) & - & Cc (Colombia) \\
\hline Isognathus scyron (Cramer, 1780) & Met & $\mathrm{Cc}(\mathrm{PNNG})$ \\
\hline Eumorpha anchemolus (Cramer, 1779) & Ant, Cnd & $\mathrm{Cc}(\mathrm{PNNG})$ \\
\hline Eumorpha phorbas (Cramer, 1775) & ¿? & ¿Cc (PNNG)? \\
\hline Xylophanes chiron nechus (Cramer, 1777) & Ant. & Cc (Colombia) \\
\hline \multicolumn{3}{|l|}{ Sphinginae } \\
\hline Agrius cingulata (Fabricius, 1775) & Ant & $\mathrm{Cc}(\mathrm{PNNG})$ \\
\hline Amphimoea walkeri (Boisduval, [1875]) & - & $\mathrm{Cc}$ (Colombia) \\
\hline Amphonyx duponchel (Poey, 1832) & ¿? & ¿Cc (PNNG)? \\
\hline
\end{tabular}

Ant: Antioquia, VC: Valle del Cauca, Snt: Santander, Met: Meta, Cc: Cauca, Cnd: Cundinamarca, ¿? No se especifica el dato. Gallego, 1946; Amarillo, 1997, 1998, 2000; Kitching, 2009; Oehlke, 2012. 

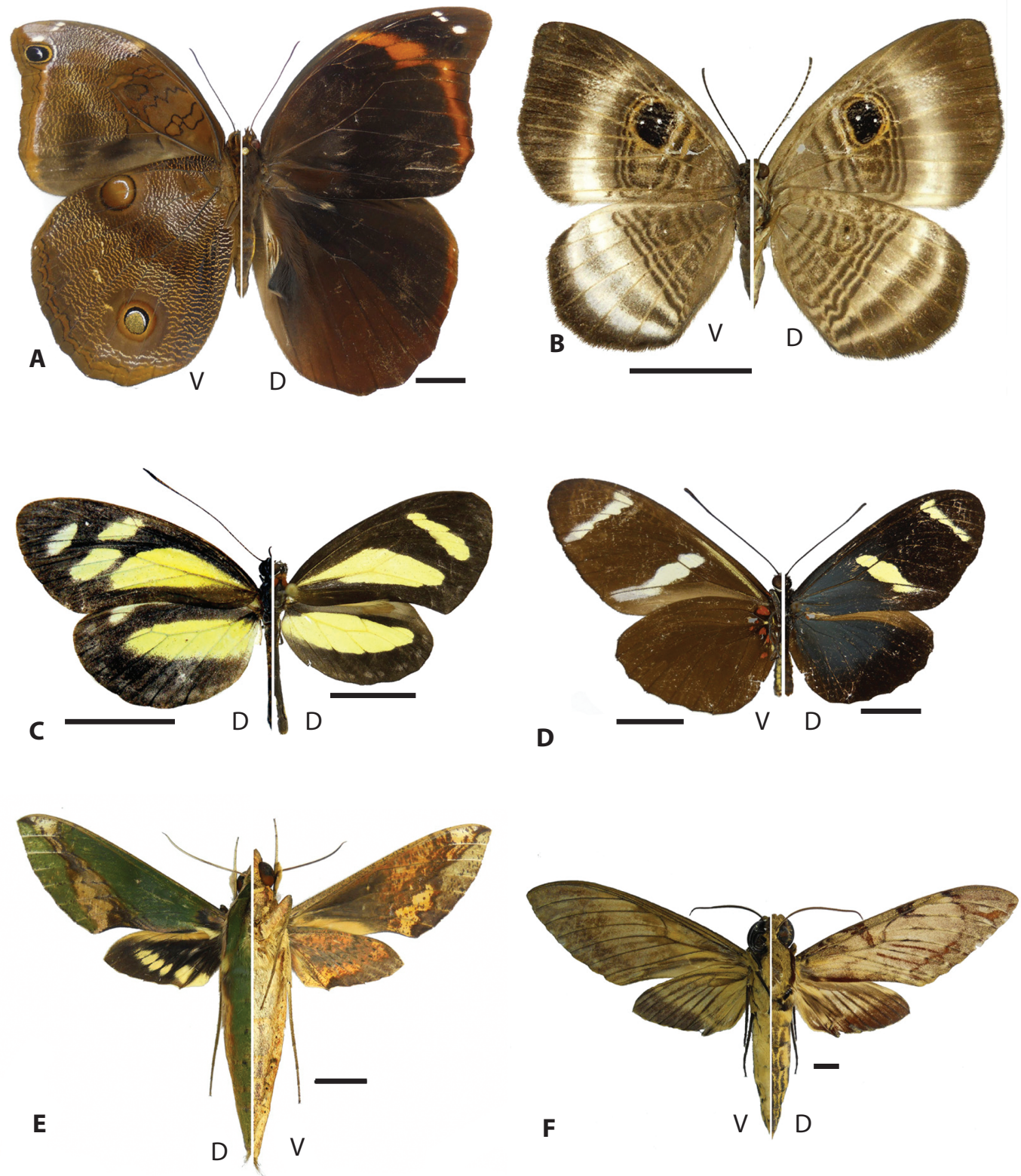

Fig. 1. Lepidópteros de PNN Gorgona. (A) Cat. xanthicles occidentalis, (B) M. zonalis gorgoniensis. (C) Mimetismo batesiano. Izquierda: Di. Theucharila avonia (copia), Derecha: Ae. Eurimedia (modelo). (D) H. Sara. (E) Amp. walkeri (F) $X$. chiron nechus. (Bar: $1 \mathrm{~cm}), \mathrm{D}=$ Vista dorsal, $\mathrm{V}=$ Vista ventral.

Fig. 1. Lepidoptera of PNN Gorgona. (A) Cat. xanthicles occidentalis, (B) M. zonalis gorgoniensis. (C) Mimetismo batesiano. Left: Di. theucharila avonia (copy), Right: Ae. eurimedia (model). (D) H. Sara. (E) Amp. walkeri (F) X. chiron nechus. (Bar: $1 \mathrm{~cm}), \mathrm{D}=$ Dorsal view, $\mathrm{V}=$ Ventral view. 
CUADRO 3

Valores de los índices de diversidad y equitabilidad para cada uno de los lugares muestreados

TABLE 3

Values of diversity and equitability indices for each of the sampled sites

\begin{tabular}{lcccc}
\multicolumn{1}{c}{ Sitio } & Perturbación & Número especies & Shannon & Equitabilidad \\
C. Trinidad & Baja & 22 & 2.45 & 0.80 \\
S. Chonta & Alta & 20 & 2.69 & 0.89 \\
P. Yundigua & Media & 17 & 2.31 & 0.81 \\
P. Blanca & Media & 16 & 2.03 & 0.76 \\
Acueducto & Media & 12 & 2.30 & 0.92 \\
Poblado & Alta & 12 & 2.21 & 0.88 \\
P. Palmeras & Baja & 9 & 1.44 & 0.62 \\
Mirador & Baja & 8 & 1.94 & 0.93 \\
\hline
\end{tabular}

de dosel no fue significativa (Spearman, $\mathrm{r}=0.82$ y $\mathrm{p}=0.1333 ; \mathrm{N}=5)$. Tampoco se detectaron diferencias significativas en la composición de especies entre los sitios muestreados (ANOSIM, $R=0.22$ y $\mathrm{p}=0.16$ ).

Distribución: Los lugares con mayor riqueza de especies fueron el Cerro Trinidad (22 especies) y Sendero de la Chonta (20 especies) y los de menor Mirador y Playa Palmeras con nueve especies cada una. El agrupamiento de los sitios según el análisis de similitud de Jaccard sugiere que la estructura del ensamble es diferente en el Poblado (Fig. 2).

Las especies con la distribución más amplia en la isla fueron: $H$. sara (Fig. 1D) (11 sitios, 166 individuos), Calycopis cerata (Hewitson, 1877) (10 sitios, 71 ind.), Ae. eurimedia (9 sitios, 72 ind.). H. sara fue más abundante entre febrero y marzo, con agregaciones de más de 25 individuos en un día.

De las especies reportadas como endémicas sólo se encontró a $M$. zonalis gorgoniensis (Fig. 1B) en Playa Yundigua y en el Poblado, camino hacia el sendero a Cerro Trinidad (Fig. 3). Se reportan, por primera vez, para la isla 14 especies de Nymphalidae, 11 de Riodinidae, tres de Lycaenidae, dos de Pieridae y una de Papilionidae. Para Saturniidae y Sphingidae, todos los registros son nuevos para la isla y dos para Colombia [Call. pan, Amp. walkeri (Fig. 1E), (Fig. 1F)]. Además, se amplía el dato de

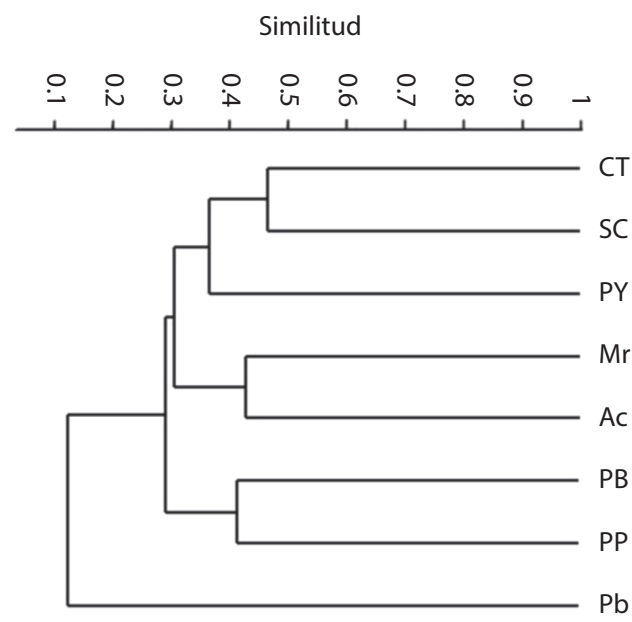

Fig. 2. Dendrograma con base en el índice de similitud de Jaccard y el algoritmo de grupos pareados de los lugares más muestreados. Convenciones: CT (Cerro Trinidad) SC (Sendero Chonta); PY (Playa Yundigua); Mr (Mirador); Ac (Acueducto); PB (Playa Blanca); PP (Playa Palmera), $\mathrm{Pb}$ (Poblado).

Fig. 2. Dendrogram based on Jaccard's similarity index and algorithm matched group of the most sampled. Conventions Convenciones: CT (Cerro Trinidad) SC (Sendero Chonta); PY (Playa Yundigua); Mr (Mirador); Ac (Acueducto); PB (Playa Blanca); PP (Playa Palmera), Pb (Poblado).

distribución para la costa Pacífica para siete de ellas (Cuadro 2).

Aspectos ecológicos: La fruta descompuesta atrajo a Eun. sydonia, Co. annulata, Ar. demophon muson, P. laertes, S. blomfildia. El pollo descompuesto atrajo a Eun. mygdonia, 


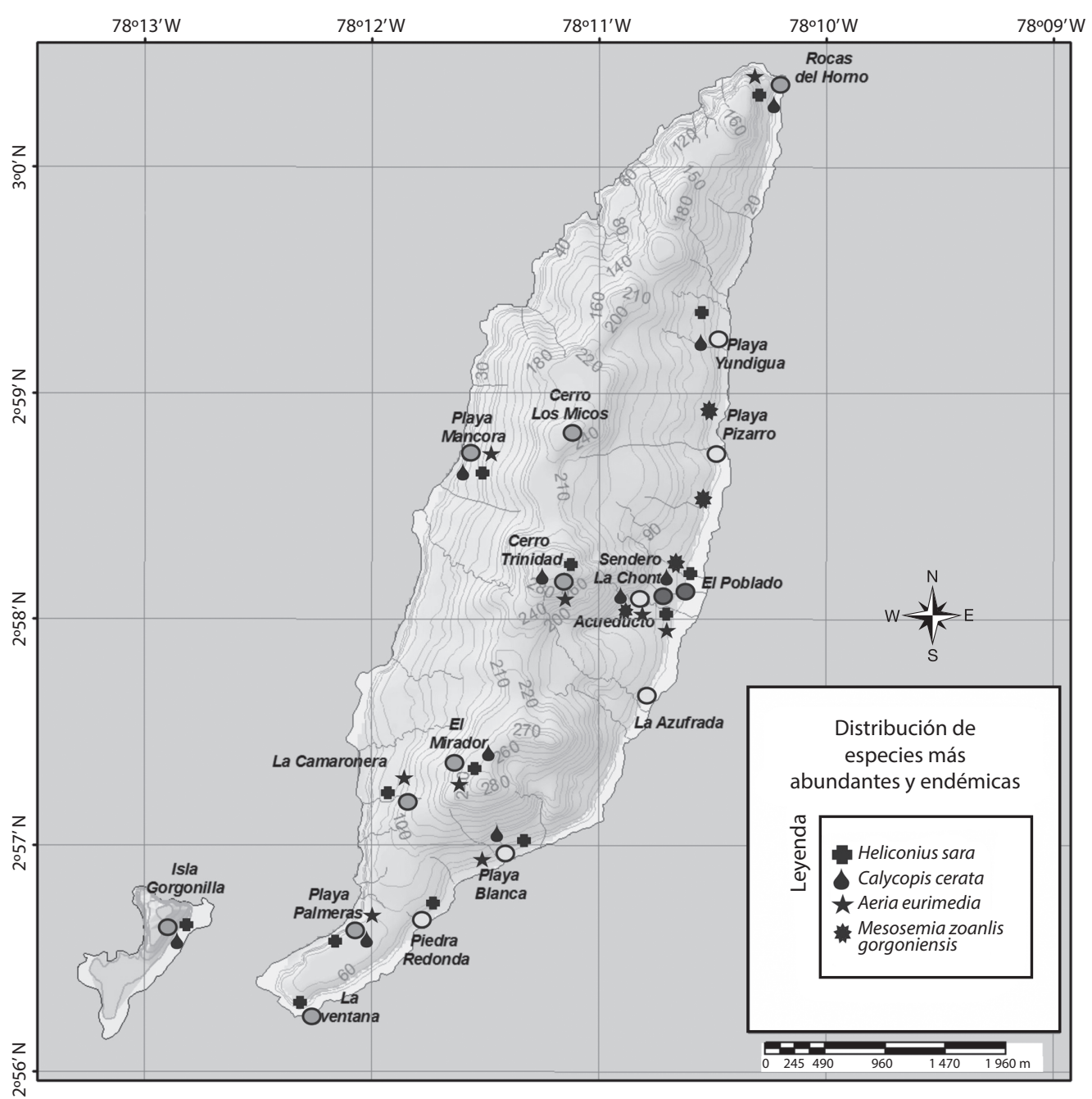

Fig. 3. Distribución de las mariposas más abundantes y endémicas del Parque Nacional Natural Gorgona.

Fig. 3. Distribution of the most abundant and endemic butterflies of Gorgona National Natural Park.

Ar. demophon musson, P. laertes. Ninguna especie fue atraída por pescado descompuesto. En trampas de luz sobre los senderos de la isla sólo se recolectaron Cal. pan y Eumorpha phorbas, el resto de especies fueron capturadas en las luces del Poblado. Los individuos de $I$. scyron se criaron desde larvas y el de $A$. cingulata fue encontrado parcialmente depredado, sin vida y con las alas intactas.

Entre las interacciones ecológicas de las mariposas de Gorgona, se observó un patrón de mimetismo batesiano entre Ae eurimedia y $D i$. theucharila avonia (Fig. 1C). Las principales plantas nectaríferas fueron: Inga punctata Willd, Palicourea triphylla DC, Psychotria tomentosa (Oerst.) Hemsl., Lantana camara Linneo, Wedelia brasiliensis (Spreng.) S.F.Blake. En plantas hospederas solo se encontró Passiflora auriculata para H. sara, Allamadra cathartica Linneo. para I. scyron. Además, se observó varios metros mar adentro a Dr. iulia y $H$. sara en vuelo desde Gorgona a Gorgonilla y viceversa. 


\section{DISCUSIÓN}

Los valores de las curvas de rarefacción, sugieren que, con excepción de El Mirador, la riqueza de especies es mayor a lo observado y se debe aumentar el esfuerzo de muestreo. El máximo índice de diversidad en Sendero Chonta (2.69), clasificado como perturbación alta, contrasta con el valor obtenido en Playa Palmeras (1.44), clasificado como perturbación baja. Es posible que este resultado obedezca a que los hábitats estuvieron en su mayoría contiguos a la playa. Aunque se había hipotetizado que los adultos de lepidópteros serían más ricos en hábitats de perturbación intermedia, este estudio no encontró un patrón consistente (Cuadro 3 ), por lo cual no hay evidencia que sugiera relación entre perturbación y riqueza de adultos. En la mayoría de los sitios, los valores de equitabilidad (por encima de 0.8 ) sugieren una comunidad de lepidópteros equitativamente distribuida, pero en Playa Palmeras el valor fue de 0.62 , lo que sugiere una mayor desigualdad comparado a los otros lugares, en el número de individuos de las especies representadas en ese sitio, como por ejemplo $H$. sara y Dr. iulia.

Los datos de este estudio más los registros de Andrade (1990), elevan la diversidad de lepidópteros del PNNG a 44 especies diurnas y 11 nocturnas. Este estudio incrementó en 31 especies el listado de Andrade (1990). El alto número de registros nuevos se logró, porque se muestreó alrededor de un $65 \%$ de la costa (senderos paralelos a la playa), dos senderos hacia los cerros e incursiones en los bosques.

Todos los registros de Sphingidae son nuevos para la isla y dos de ellos para el país, lo que refleja el poco estudio de la familia a nivel nacional. Amarillo (1997) reporta la región Pacífica, como la segunda más rica en diversidad de Saturniidae del país, sin embargo, en Gorgona solo se encontró a $R$. lebeau. Los Saturniidae pueden haber disminuido por la constante intervención antrópica durante la época de prisión, ya que la vertiente oriental sufrió el mayor grado de deterioro (Ortiz, 1991).
Cerro Trinidad posee una vegetación muy heterogénea, con bosques primarios (partes altas) e intervenidos (parte baja), los cuales colindan con el Sendero de la Chonta, con tan solo dos especies menos. Esta heterogeneidad favorece y puede mantener una alta diversidad de mariposas (Brown \& Freitas, 2002). Esto explica que su agrupamiento en el dendrograma fuese de $47 \%$. En el Poblado, que es el lugar más intervenido antrópicamente, se observó una clara diferencia con los demás lugares de alrededor del 90\%. Sin embargo, fue notoria la presencia de varias especies que son comunes tanto en ambientes conservados como perturbados y urbanos (Ramírez, Chacón de Ulloa \& Constantino, 2007; Lazzeri, Bar \& Pieri-Damborsky, 2011).

La especie más abundante en la isla fue $H$. sara. Los individuos de esta especie forman agregaciones, alrededor de 25 , con fines reproductivos y de ovoposición. Estos grupos se observan cuando cuando su hospedero, Pas. auriculata es abundante (Gilbert, 1991 citado por Ramírez, 2007). Calycopis cerata y Ae. eurimedia también tuvieron amplia distribución y abundancia, con más frecuencia en microhábitats con sombra. El pollo descompuesto atrajo algunas mariposas como $P$. leartes, Eun. sydonia y Eun. mygdonia que son especies de vuelo alto y veloz que rara vez bajan al suelo (DeVries, 1987).

El mimetismo batesiano entre Ae. eurimedia (modelo) y Di. theucharila avonia (copia) se basa en un mecanismo de defensa visual química para advertir a potenciales depredadores (Arango, 2005). En pleno vuelo parecen la misma especie, sin embargo Ae. eurimedia presenta vuelo más pausado y prefiere el sotobosque a diferencia de Di. theucharila avonia, la cual vuela en sotobosque y escapa rápidamente a mas altura (H. Calero, obs. pers.).

Se deben diseñar estudios (captura-recaptura) para conocer mejor la dispersión de las especies. Se observó a $H$. sara volando hacia Gorgonilla, en actividades de cópula y ovoposición y a Dr. iulia en mar abierto (H. Calero obs. pers.), lo que permite especular sobre la posible migración de individuos adultos 
desde el continente hacia la isla y viceversa. Isla Gorgona está a solo $35 \mathrm{~km}$, en línea recta del continente (Yockteng \& Cavelier, 1998), distancia que fácilmente es superada por especies de largo vuelo como la familia Uranidae, ampliamente migratoria de la costa Pacífica y encontrada en Gorgonilla.

La correlación entre la cobertura vegetal de dosel y la riqueza de mariposas no fue significativa, pero se suelen registrar más especies en mayores porcentajes de cobertura de dosel (entre 80 y 100\%). Es posible que este resultado se deba a que la mayoría de los senderos muestreados están en proceso de regeneración. La cobertura de dosel regula la cantidad de luz que entra al bosque (Whitmore, 1998) y es un factor importante para insectos ectotérmicos, como las mariposas, que puede disminuir la riqueza en zonas muy cubiertas. Sin embargo la presencia de adultos en bosque maduro se puede explicar en términos de recursos e historia natural. Por ejemplo, Gilbert (1980) reporta que algunos machos adultos de Ithomiinae requieren recursos del bosque maduro para necesidades metabólicas y de cortejo. Por otro lado, algunas especies aprovechan pequeñas cantidades de luz o vuelan hacia ramas altas para calentarse (H. Calero obs. pers.). La cantidad de luz puede modelar la estratificación (DeVries, 1987) e incluso la diversidad de mariposas como ocurre con las especies de Ithomiinae, Brassolinae y Satyrinae, especialistas de microhábitats sombreados; mientras que las Heliconiinae, Nymphalinae y Riodinidae, pueden moverse entre lugares soleados y sombreados (Valencia et al., 2005).

Este estudio contribuyó al incrementar la lista de especies diurnas publicadas para la isla en más del doble además de suministrar los primeros reportes para polillas, información que se constituye en una línea de referencia para posteriores estudios que permitirá fortalecer las acciones de conservación del PNN Gorgona.

\section{AGRADECIMIENTOS}

A Hans Dahners, Carlos Prieto, Keith Willmot, Cristobal Ríos, Bill Oehlke, Ángela
Amarillo, Patrick Blandin, y Lorena Ramírez por confirmar la identificación taxonómica de las especies, y los valiosos comentarios realizados a una versión preliminar de este manuscrito. A Ranulfo González, María del Carmen Zuñiga, Alan Giraldo, Marcela González, Diana Torres, Julián Mendivil y Stephanie Valdés, por su colaboración en campo, sus consejos y aportes promovieron la buena interpretación de los datos. A Ximena Urrutia por la ayuda en los análisis de datos. Este trabajo fue financiado parcialmente por el Fondo para la Acción Ambiental y la Niñez, Conservación Internacional Colombia, Universidad del Valle y la Fundación Squalus, y se realizó en el marco del proyecto de investigación "Evaluación del estado actual de los objetos de conservación faunísticos en isla Gorgona: una aproximación holística a la valoración ecológica de PNN Gorgona", amparado por el permiso de investigación PIBD-DTSO 011-10. La Fundación Samanea, Fundación Bioethos (Colombia) e Idea Wild (EE.UU.) que apoyaron económicamente la finalización de esta investigación. A los evaluadores anónimos por sus valiosos comentarios y aportes que permitieron enriquecer este manuscrito.

\section{RESUMEN}

Gorgona es una isla ubicada en la costa Pacífica de Colombia. Durante 26 años fue una prisión, pero desde 1986 fue declarada Parque Nacional Natural, y desde entonces se encuentra en un proceso de sucesión natural. En este estudio se presenta una lista de los lepidópteros de isla Gorgona y se analizan algunos de los ecológicos que podrían promover la biodiversidad in situ de este grupo taxonómico. Se recolectaron mariposas y polillas durante tres viajes realizados entre octubre de 2010 y mayo de 2011. Las observaciones de las mariposas se realizaron a lo largo de los principales senderos de la isla. Se instalaron trampas cebadas con fruta, pescado y pollo descompuestos, durante tres días en cada zona de trabajo (dos tipos cebos por sitio) como también capturas con redes de entomológicas entre las 09:00h y 18:00h. En el muestreo de las polillas se utilizaron trampas de luz blanca y luz negra en zonas abiertas, la luz se reflejó sobre una pantalla blanca para maximizar la atracción. Además, se realizaron revisiones diarias en los alrededores de las luces artificiales del poblado. Se registró la cobertura del dosel del bosque en cada punto de muestreo. En total, se identificaron 52 
especies pertenecientes a siete familias, de las cuales 31 de mariposa y 11 de polillas fueron nuevos registros para la isla. El Cerro Trinidad y el Sendero de la Chonta fueron los sitios de muestreo con mayor riqueza de especies (22 y 20 , respectivamente). La riqueza de especies de mariposas no disminuyó con la reducción de la cobertura del dosel. Heliconious sara y Aeria eurimedia fueron las especies dominantes en número, pero su distribución no se superpuso. Tres especies fueron las más ampliamente distribuidas en la isla: H. sara (11 siitios, 166 individuos), Calycopis cerata (10 sitios y 71 individuos) y Ae . eurimedia (9 sitios y 72 individuos). Se reporta Catoblepia xanthicles occidentalis después de 87 años. La mayoría de las curvas de acumulación de especies no fueron asintóticas lo que sugiere que la diversidad de la isla es más alta de lo esperado. Este estudio contribuyó a incrementar en más del doble el listado de especies de mariposas reportadas para isla Gorgona y contiene los primeros registros de Polillas para esta localidad.

Palabras clave: Colombia, mariposas, inventario, polillas, parque nacional, isla.

\section{REFERENCIAS}

Aldrete, A. N., González, O. R. \& Sarria, F. A. (2011). Three new species of Loneura (Psocodea: "Psocoptera": Ptiloneuridae) from Gorgona Island, Cauca, Colombia, with a new infrageneric classification. Zootaxa, 3050: 55-62.

Amarillo, A. R. (1997). Satúrnidos de Colombia (Lepidoptera: Saturniidae) (excepto Hemileucinae) (Tesis de Maestría). Universidad Nacional de Colombia, Colombia.

Amarillo, A. R. (1998). Uso de polillas (lepidóptera: Heterocera) como bioindicadores del estado de conservación del ecosistema (Informe final). Fundación para la promoción de la investigación y la tecnología, Santa Fé de Bogotá, Colombia.

Amarillo, A. R. (2000). Polillas Satúrnidas (Lepidoptera: Saturniidae) de Colombia. Biota Colombiana, 1: 177-186.

Amat, G. G., Andrade, M. G. \& Amat, G. E. (2007). Libro Rojo de invertebrados terrestres de Colombia. Bogotá. Instituto de Ciencias Naturales, Universidad Nacional de Colombia, Conservación Internacional Colombia, Instituto Alexander von Humboldt, Ministerio de Ambiente, Vivienda y Desarrollo Territorial, Bogotá, Colombia.

Andrade, G. (1990). Inventario de mariposas (Lepidoptera: Rhopalocera). In J. Aguirre \& O. Rangel (Eds.), Biota y Ecosistemas de Gorgona (pp. 252-254) Fondo FEN, Bogotá, Colombia.

Arango, L. (2005). Identificación de las especies miméticas de mariposas en la Reserva Natural Karagabí y el jardín botánico de Pueblo Rico (Pueblo Rico, Risaralda, Colombia) parte I. Boletín de la Sociedad Entomológica Aragonesa, 36: 303-307.

Baena, M. L. \& Alberico, M. (1991). Relaciones biogeográficas de las hormigas de la Isla Gorgona. Revista Colombiana de Entomología, 17: 24-31.

Baena, M. L. (1992). Relaciones biogeográficas de las hormigas de Isla Gorgona (Trabajo de pregrado). Universidad del Valle, Santiago de Cali, Colombia.

Brown, K. S. (1991). Conservation of neotropical environments insects as indicators. p. 349-404 In N. M. Collins \& J. A. Thomas (Eds.), The conservation of insects and their habitats. Royal Entomology Society Symposium XV, Londres, England.

Brown, K. S. \& Freitas, A. V. L. (2002). Butterfly communities of urban forest fragments in Campinas, São Paulo, Brazil: structure, instability, environmental correlates, and conservation. Journal of Insect Conservation, 6: 217-231.

D'Abrera, B. (1987). Butterflies of the Neotropical Region. Part IV. Nymphalidae (partim). Hill House. Victoria. Black Rock.

D'Abrera, B. (1988). Butterflies of the Neotropical Region. Part V. Nymphalidae (Conc.) \& Satyridae. Hill House, Black Rock, Victoria, Australia.

D'Abrera, B. (1994). Butterflies of the Neotropical Region. Part VI. Riodinidae. Hill House, Victoria, Australia.

D'Abrera, B. (1995). Butterflies of the Neotropical Region. Part VII. Lycaenidae. Hill House, Victoria, Australia.

DeVries, P. J. (1987). The butterflies of Costa Rica and their natural history (Papilionidae, Pieridae, Nymphalinae). Princeton University, Nueva Jersey, USA.

Gallego, F. L. (1946). Catálogo de Insectos determinados correspondientes al Orden Lepidoptera, existentes en la sección de Entomología de la Facultad de Agronomía. Revista de la Facultad de Agronomía de Medellín, 6(24): 415-473.

Gema Virtual. (2007). Banco de imágenes, Unidad de Producción Audiovisual (UPA). Instituto de Investigación de Recursos Biológicos Alexander von Humboldt, Bogotá, Colombia. Retrieved from http://www. humboldt.org.co/chmcolombia/servicios/jsp/galeria/

Gilbert, L. E. (1980). Food web organization and conservation of neotropical diversity. In M. E. Soule \& B. A. Wilcox (Eds.), Conservation Biology: an evolutionary-ecological perspective (pp. 11-34). Sinauer Associates, Inc. Sunderland, Massachusetts, USA.

Gilbert, L. E. (1991). Biodiversity of a Central American Heliconius community: pattern, process, and problems. In P. W. Price, T. M. Lewinsohn, G. W. Fernandes \& W. W. Benson (Eds.), Plant-animal Interactions: Evolutionary Ecology in Tropical and temperate Regions (pp. 403-427). New York, USA. 
Gómez, A. (2009). Variación de la estructura y composición de los ensamblajes de macroinvertebrados bénticos en la jerarquía espacial de los sistemas lóticos del PNN Gorgona (Trabajo de pregrado). Universidad de Antioquia, Medellín, Colombia.

González, R. \& Sarria, F. (2010). Informe Preliminar de la expedición científica entomológica al PNN Isla Gorgona. Universidad del Valle, Santiago de Cali, Colombia.

Green, D. G., Sadedin, S. \&. Leishman, T. G. (2009). Self organization. In S. E. Jorgensen (Ed.), Ecosystem Ecology (pp. 98-106). Elsevier, Amsterdam, Netherlands.

Hammer, O., Harper, D. A. T. \& Ryan, P. D. (2008). PASTPalaeontological Statistic, ver. 1.77. Retrieved from http://www.folk.unio.no/ohammer/past

Kattán, G. H. \& Naranjo, L. G. (2008). Regiones biodiversas: herramientas para la planificación de sistemas regionales de áreas protegidas. Fundación EcoAndina, WCS-Colombia, WWF-Colombia, Cali, Colombia.

Kitching, I. J., Scoble, J. M., Smith, C. R., James, S., Young, R. \& Blagoderov, V. (2009). CATE Sphingidae. Retrieved from http://www.cate-sphingidae. org? view=33233133-33be-462e-a10d-31b5fd24748e

Lamas, G. (2004). Checklist: Part 4A. Hesperioidea-Papilionoidea. In J. B. Heppner (Ed.), Atlas of Neotropical Lepidoptera (Vol. 5A). Association for Tropical Lepidoptera. Scientific Publishers, Gainesville, USA

Lazzeri, M. G., Bar, M. E. \& Pieri-Damborsky, M. (2011). Diversidad del orden Lepidoptera (Hesperioidea y Papilionoidea) de la ciudad Corrientes, Argentina. Revista de Biología Tropical, 59(1): 299-308.

Le Crom, J. F., Constantino, L. M. \& Salazar, J. A. (2002). Mariposas de Colombia. Tomo I: Papilionidae. Carlec LTDA, Colombia.

Oehlke, B. (2012). Sphingidae of the Americas. Retrieved from http://www.silkmoths.bizland.com/danjansphinx.htm

Ortiz, B. (1991). Caracterización biográfica y ecológica de las aves terrestres de la isla Gorgona (Trabajo de grado). Universidad del Valle, Cali, Colombia.
Pimienta, C., Montealegre, F., López-Mejía, J., Chacón de Ulloa, P. \& González- Obando, R. (2008). Diversidad de artrópodos arbóreos en el PNN Gorgona (Cauca, Colombia). In Sociedad Colombiana de Entomología. Resúmenes Congreso de la Sociedad Colombiana de Entomología, Colombia.

Prieto, C. \& Dahners, H. (2006). Eumaeini (Lepidoptera: Lycaenidae) del cerro San Antonio: Dinámica de la riqueza y comportamiento de "Hilltopping". Revista Colombiana de Entomología, 32 (2): 179-190.

Ramírez, R. L., Chacón de Ulloa, P. \& Constantino, L. M. (2007). Diversidad de mariposas diurnas (Lepidoptera: Papilionoidea y Hesperioidea) en Santiago de Cali, Valle del Cauca, Colombia. Revista Colombiana de Entomología, 33: 54-63.

Salazar, J., Rodríguez, G. \& Constantino, L. (2009). Contribución al conocimiento del género Mesosemia Hübner [1819] en Colombia y descripción de nuevos taxa (Lepidoptera: Riodinidae). Boletín Cientifico Museo de Historia Natural, 13(2): 174-240.

Torres, O., Pinilla, G. \& Chamorro, C. (1998). Artropofauna de los suelos de Isla Gorgona. Colombia Geográfica, 14: 22-29.

Valencia, C., Gil, Z. \& Constantino, L. M. (2005). Mariposas diurnas de la zona central cafetera de Colombia. Cenicafé, Chinchiná, Colombia.

Villareal, H., Álvarez, M., Córdoba, S., Escobar, F., Fagua, G., Gast, F., Mendoza, H., Ospina, M. \& Umaña, A. M. (2004). Manual de métodos para el desarrollo de inventarios de biodiversidad. Programa de inventarios de biodiversidad. Instituto Alexander von Humboldt, Bogotá, Colombia.

Warren, A. D., Davis, K. J., Grishin, N. V., Pelham, J. P. \& Stangeland, E. M. (2011). Interactive Listing of American Butterflies. The butterflies of americas foundation. Retrieved from http:// www.butterfliesofamerica.com

Whitmore, T. C. (1998). Introduction to rain forest. Oxford University, England.

Yockteng, R. \& Cavelier, J. (1998). Riqueza, diversidad y mecanismos de dispersión de árboles de la isla Gorgona y de los bosques húmedos tropicales del pacífico Colombo-Ecuatoriano. Revista de Biología Tropical, 46(1): 45-53. 